\title{
Antimicrobial Photodynamic Therapy for Treatment of Refractory Chronic Rhinosinusitis: A Pilot Study
}

Luis Macias Valle, Andres Finkelstein Kulka, Saad Alsaleh, Christopher Okpaleke, Jamil Manji, Fahad Alasousi and Amin Javer*

St. Paul's Sinus Center, St. Paul's Hospital, Vancouver, Canada

*Corresponding author: Amin Javer, St. Paul's Sinus Center, St. Paul's Hospital,

Vancouver, Canada.

\section{Abstract}

Background: Inflammation in refractory chronic rhinosinusitis (RCRS) is complicated by persistent microbial colonization resistant to medical treatment. Antimicrobial photodynamic therapy (aPDT) has been shown to be effective in eradicating in-vitro biofilms of chronic rhinosinusitis microbes and reducing mucosal inflammation. It is an emerging tool for treatment of RCRS.

Objective: To evaluate the safety and efficacy of aPDT in a cohort of our patients diagnosed with RCRS.

Study design: Retrospective case series.

Materials and methods: Patients with persistent inflammation and suspected chronic biofilm within the sinuses despite appropriate medical and surgical treatment were treated with aPDT using a diode laser and methylene blue (as photosensitizer) on an outpatient basis. Patient outcomes were reviewed at 3 and 6 months post-aPDT. Endoscopic sinus scores and adverse events were recorded at each visit.

Results: Sixteen patients were recruited and treated with aPDT. Of these, 14 patients completed their follow-up visits. Forty-three sinuses (5-frontal, 21-ethmoid, 16-maxillary, 1-sphenoid) in the fourteen patients (13-females, 1-male) were treated (Mean: 2.7 sinuses/patient per treatment). The average age of patients was 53.7 years. Nine of the fourteen patients treated showed improved endoscopic scores in the sinuses after 6 months. (Mean MLK Score difference \pm SD: 1.81 \pm 2.76 ). Three patients expressed minor and transient adverse events (slight bleeding, stinging sensation) immediately after the procedure and none at 3 and 6 months. The clinicians' experience with the procedure was satisfactory.

Conclusion: Patients with CRS can be safely treated with aPDT on an outpatient basis. These early results, while promising, will require validation in prospective clinical trials.

Keywords: Refractory Chronic Rhinosinusitis; Photodynamic therapy; Biofilm; Methylene blue

\begin{abstract}
Abbreviations: aPDT: Antimicrobial photodynamic therapy; AFRS: Allergic fungal rihinosinusitis; CRS: Chronic rhinosinusitis; CRSwNP: Chronic rhinosinusitis with nasal polyps; CRSsNP: Chronic rhinosinusitis without nasal polyps; EDTA: Ethylenediaminetetraacetic acid; FESS: Functional endoscopic sinus surgery; MAD: Mucosal atomization device; MLK: Modified Lund-Kennedy; MRSA: Methicillin-resistant Staphylococcus aureus; QoL: Quality of life; RCRS: Refractory chronic rhinosinusitis
\end{abstract}

\section{Introduction}

Rhinosinusitis is among the most common conditions in North America, affecting more than 31 million people annually [1,2]. Chronic rhinosinusitis (CRS) prevalence, however, is difficult to extrapolate because of the heterogeneity of its presentation and imprecise diagnosis [3]. Refractory chronic rhinosinusitis (RCRS) is defined as persistence of signs and symptoms of CRS despite technically adequate functional endoscopic sinus surgery (FESS) and adequate postoperative medical management [4]. RCRS has significant detrimental health effects such as reduced quality of life (QoL), fatigue, sleep disturbance, sexual dysfunction, olfactory dysfunction 
and depression [5]. The summation of these effects has a profound impact on productivity. It is estimated that the annual productivity cost associated with RCRS is $\$ 10,077.07$ per patient [6].

Several pathophysiologic mechanisms for persistent CRS disease have been described. One of these is persistent bacterial biofilm formation secondary to a microbial dysbiosis within the affected sinonasal cavity [7]. Biofilms are a complex organized community of germs that adhere to the mucosal surface and surrounded by an extensive extracellular polymeric substance (glycocalyx) that is composed primarily of polysaccharides [8]. They begin their life cycle as independent bacteria, which become sessile and initiate the biofilm formation process by adhering to a surface and forming microcolonies [9]. Biofilms allow bacteria to evade host defenses and decrease susceptibility to antibiotic therapy. They also cause a deliberate release of planktonic bacteria, resulting in implantation and population of new anatomic locations [10]. Several organisms have been implicated in the formation of biofilms including: Staphylococcus aureus, Pseudomonas aeruginosa, Streptococcus pneumoni$a e$ and other gram-negative rods [11]. Due to the unique properties of biofilms, new directions in therapy are constantly being sought for the treatment of patients with RCRS secondary to microbial dysbiosis and biofilm formation [12].

Antimicrobial photodynamic therapy (aPDT) is a novel treatment modality showing promise in the treatment of bacterial biofilms. It has been applied successfully in dentistry [13], wound infections [14], oncology [15,16], dermatology [17] and gynecology [18]. A range of photosensitizing molecules have been used with phenothiazinium dyes (methylene blue, toluidine blue, and PP904) being the most common [19]. Investigations of aPDT in vitro have shown it to be lethal against all classes of microorganisms: gram-positive and gram-negative bacteria, fungi, viruses, parasites and even spores [20]. In addition to its antimicrobial effects, PDT also demonstrates a marked anti-inflammatory effect, and has also been shown to effectively treat the polymicrobial biofilms involved in CRS [21-23]. We describe a pilot study on the safety and efficacy of aPDT in the management of CRS patients who are refractory to appropriate medical and surgical therapy.

\section{Materials and Methods}

\section{Patients}

Ethics approval for this study was obtained from the Providence Health Care Research Ethics Board of the University of British Columbia (Ethics number: H15-01411). Electronic medical charts were reviewed of all patients treated with aPDT between November 2013 and March 2015 at our tertiary rhinology centre.

Patients were included in this review if they had undergone at least one aPDT treatment at our centre. Patients are only offered aPDT if they had already received complete FESS at least 6 months prior and also demonstrated recalcitrance to appropriate adjunctive medical therapy. Appropriate adjunctive medical therapy at our centre includes at least 18 weeks of topical budesonide (Pulmi- $\operatorname{cort}($, Astra Zeneca,) $0.5 \mathrm{mg} / 2 \mathrm{ml}$ delivered via impregnated nasal saline irrigation or the mucosal atomization device (MAD), 3 weeks of culture-directed oral antibiotics or antifungals (Itraconazole) as necessary for more than 2 discrete infections per year, at least 6 weeks of low-dose macrolide therapy, and oral steroids (Prednisone $0.5 \mathrm{mg} / \mathrm{kg} 2$ week taper).

Patients with sinonasal tumors, autoimmune and systemic inflammatory diseases affecting the upper airway were excluded. Patients receiving aPDT were followed up regularly post-aPDT with detailed endoscopic examinations and graded using the modified Lund-Kennedy (MLK) endoscopic scoring system. The scores were recorded at baseline and at each follow-up visit after aPDT treatment. Digital images of the sinuses were recorded at each visit. Adverse events reported in the immediate post-procedure period were also recorded.

\section{Materials and treatment procedure}

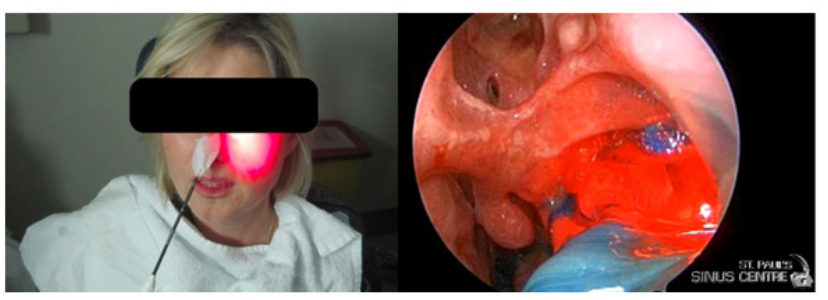

Figure 1: (Left): Patient undergoing left maxillary sinus photodynamic therapy. The illumination of the sinus is visualized. (Right): Endoscopic view of the left maxillary sinus with the balloon catheter inserted, prior application of methylene blue.

The device used for the aPDT was the SinuwaveTM Ceralas E® (Ondine Biomedical). Following endoscopy, nasal debridement was performed if needed and bacterial sinus culture was collected via a culture swab (Starswab II ${ }^{\circledR}$, Starplex Scientific Inc., Ontario, Canada) or specimen trap (Argyle ${ }^{\mathrm{TM}}$ Covidien, MA, USA). Three milliliters of the photosensitizing agent (methylene blue) was then sprayed into the affected sinuses using a curved suction under direct endoscopic visualization with a $3 \mathrm{~mm} 30^{\circ}$ rigid endoscope. The Sinuwave Light Delivery Catheter (Figure 1) is made of a malleable yet firm material that is bent to facilitate the introduction into the sinus to be treated. The distal end of the catheter houses a balloon that surrounds the laser projecting tip. Inflation of the balloon facilitates equivocal assortment of the photosensitizing agent on the sinus wall mucosa, mechanical evacuation of purulent or mucinous discharge that is located in the sinus as well as equal distribution of the laser light to the surrounding mucosa. The catheter is introduced under endoscopic guidance, occasionally under topical anesthesia (Lidocaine $10 \%$ spray, $20 \mathrm{mg}$ per nostril), into the sinus that is about to undergo the treatment. The balloon is inflated with saline. The amount of saline used is determined by the volume of the sinus cavity. As the balloon inflates, it starts to swell in a retrograde fashion out of the ostium and the resistance to balloon inflation increases. The inflation is carried out via a port located at the proximal end of the catheter. The catheter is connected to the 
Sinuwave Laser Console. Once in place and well inflated, the sinus is illuminated using a low-level laser at $670 \mathrm{~nm}$ wavelength (Figure $1)$. The laser power ( $0.5 \mathrm{~W}$ to $4.90 \mathrm{~W})$ is calibrated according to the amount of saline $(<1 \mathrm{ml}-15 \mathrm{ml})$ used to inflate the balloon, as established by the manufacturers protocol. Once activated, the catheter is left in place for a duration of 4 minutes. At the conclusion of treatment, the balloon is deflated and removed. The sinus cavity is then re-examined and evaluated for any immediate adverse reactions to the treatment.

\section{Statistical analysis}

Demographic and baseline characteristics were extracted from patient charts and recorded for each subject. Demographic data included age (years), sex, history of smoking and ethnicity. Baseline characteristics included diagnosis, history of sinus surgery, endoscopic sinus score (MLK), endoscopic imaging, and culture results collected prior to receiving aPDT treatment. The number of sinuses treated per session was also recorded. Categorical, explanatory variables were summarized by frequency and absolute proportions. Continuous, explanatory variables were summarized by mean and standard deviation. The primary outcome variable was endoscopic mucosal scores evaluated before and after treatment. The outcome variable was recorded as a continuous, numerical outcome and summarized by mean and standard deviation. Data

Table 1: Subject demographics and baseline clinical characteristics.

\begin{tabular}{|c|c|c|}
\hline Characteristics & Mean (SD) & Frequency (\%) \\
\hline Age (years) & $53.7(13.4)$ & \\
\hline $\begin{array}{c}\text { Sex: } \\
\text { Female } \\
\text { Male }\end{array}$ & & $\begin{array}{c}13(93 \%) \\
1(7 \%)\end{array}$ \\
\hline $\begin{array}{c}\text { Race: } \\
\text { Caucasian } \\
\text { South Asian }\end{array}$ & & $\begin{array}{l}12(86 \%) \\
2(14 \%)\end{array}$ \\
\hline $\begin{array}{c}\text { Smoking History: } \\
\text { Current/Previous smoker } \\
\text { Never smoked }\end{array}$ & & $\begin{array}{c}0(0 \%) \\
14(100 \%)\end{array}$ \\
\hline $\begin{array}{l}\text { Diagnosis: } \\
\text { CRSwNP } \\
\text { AFRS } \\
\text { CRSsNP }\end{array}$ & & $\begin{array}{c}11(79 \%) \\
9 / 11(64 \%) \\
3(21 \%)\end{array}$ \\
\hline Number of Surgeries (FESS) & $1.14(0.4)$ & \\
\hline Number of aPDT Treatments & $2.7(1.5)$ & \\
\hline $\begin{array}{c}\text { Pre-aPDT Sinus Cultures: } \\
\text { Fungi (Aspergillus, Rhizopus) } \\
\text { Gram positive cocci (S. aures, S. epidermidis, S. pnemoniae) } \\
\text { Gram negative bacilli (H. Influenzae, Klebsiella, E. coli) }\end{array}$ & & $\begin{array}{l}5(42 \%) \\
6(50 \%) \\
3(21 \%)\end{array}$ \\
\hline
\end{tabular}

aPDT: Antimicrobial photodynamic therapy; AFRS: Allergic fungal rhinosinusitis; CRSwNP: Chronic rhinosinusitis with nasal polyps; CRSsNP: Chronic rhinosinusitis without nasal polyps; FESS: Functional endoscopic sinus surgery; SD: Standard deviation.

were collected, de-identified, and stored in a securely encrypted electronic database.

\section{Results}

Sixteen patients were recruited and treated with aPDT at our centre during the review period (November 2013 to March 2015). All of the demographic data is summarized in (Table 1). Of these, 14 patients were followed up for at least 6 months after the procedure. Two patients did not complete 3 months of follow up, however they were followed up at 6 months and no adverse effects were noted. Clinical and baseline characteristics are summarized in (Table 1). Two patients received 2 successive aPDT treatments while the others $(n=12)$ received only one treatment. Forty-three sinuses (5-frontal, 21-ethmoid, 16-maxillary, 1-sphenoid) in the fourteen patients were treated (Mean: 2.7 sinuses/patient per treatment with a total of 16 treatments). Eleven (79\%) of the patients were being managed for chronic rhinosinusitis with nasal polyps (CRSwNP) and 3 (21\%) were managed for chronic sinusitis without nasal polyps (CRSsNP). Nine (64\%) of the patients had also been diagnosed with allergic fungal rhinosinusitis (AFRS) using the Bent and Kuhn diagnostic criteria [24]. The average number of FESS procedures undergone by the patients prior to their initial aPDT session was $1.14 \pm 0.4$ (mean $\pm S D$ ). 
Table 2: Mean endoscopic sinus scores pre and post aPDT.

\begin{tabular}{|c|c|}
\hline & Modified Lund-Kennedy (MLK) Score Mean (SD) \\
\hline Baseline Pre aPDT & $3.75(2.1)$ \\
\hline 3 months post aPDT & $1.37(1.6)$ \\
\hline 6 months post aPDT & $1.94(1.8)$ \\
\hline
\end{tabular}

aPDT: Antimicrobial photodynamic therapy; SD: Standard deviation.

Nasal cultures done prior to the aPDT showed various classes of microbes; fungi (Aspergillus sp. and Rhizopus), gram-positive cocci (S. aureus, S. epidermidis and S. pneumoniae) and gram-negative bacilli (H.influenzae, Klebsiella and E.coli). The mean difference ( \pm standard deviation) in the pre and post endoscopic scores was $2.38 \pm 2.96$ (range: $-2-34$ points) using the MLK scoring system. Endoscopic sinus scores taken at 3 and 6 months showed a reduction in inflammation from baseline in 9 of the 14 patients (Table 2). Regarding safety of the procedure, no major complications were observed. Out of 43 sinuses intervened, 3 reports of transient effects were identified. The adverse events included slight bleeding, stinging sensation in the sinuses and a slight headache during the procedure. None of these events required medical intervention or persisted for any length of time beyond the clinic visit.

\section{Illustration: case $\mathrm{E}$}

An 89-year-old male being managed for RCRS with nasal symptoms for over 2 years. Current treatment included topical intranasal steroids, saline irrigation, multiple episodes of long-term low dose macrolide antibiotics and oral steroids. He presented with a history of recurrent sinus infections, nasal polyposis, hyposmia and long-standing nasal obstruction. He underwent FESS 9 months prior to his first aPDT procedure. Intra-operative findings revealed multiple grade 3 polyposis and purulent discharge in all sinuses. His immediate postoperative management was uneventful. Post-operatively, he began to suffer from recurrent episodes of sinus infections. He continuously cultured S. aureus from his sinuses at every visit despite antibiogram guided oral and topical antibiotic and anti-inflammatory treatment for his CRS.

He received bilateral aPDT to all sinuses, and showed an immediate improvement in endoscopic sinus scores. His MLK mucosal grading score improved from a total of $4 / 6$ on the right side to $0 / 6$ at three months follow-up. However, his left side was at $0 / 6$ and showed mild inflammation of $2 / 6$ at three months follow-up. He then received a second treatment of aPDT 6 months later due to the suboptimal response. At that time his right side remained stable with mild edema (1/6) and the left side scores were 4/6 immediately prior to the second aPDT treatment. This dropped to $2 / 6$ three months post-treatment. Post aPDT sinus culture showed normal respiratory flora. His medical management remained the same 6 months prior to aPDT and 6 months post aPDT.

\section{Discussion}

Treatment and management of refractory CRS continues to be a difficult scenario for rhinologists. Despite adequate surgery and medical treatment, gaps in current knowledge about etiology and disease characterization, clinical control in this group of patients is challenging. Different agents have been described as possible causatives including chronic osteitis [25], chronic bacterial infection, fungi [26] and staphylococcal superantigens.

Photodynamic therapy is a non-invasive non-pharmacological treatment, with widespread use in other fields of medicine [1315,17]. In chronic periodontal disease aPDT has demonstrated efficacy in cases where conventional antibiotic therapies can be challenging (ie. biofilms, gram-negative bacteria, and antimicrobial resistant organisms). The mechanism of action is based on the concept of disrupting the bacterial cell wall structure with oxygen free radicals. These free radicles are generated as a result of the interaction between certain wavelengths of laser light and photoactive material such as methylene blue dye in our study. Several studies have shown promising results in reduction of methicillin-resistant Staphylococcus aureus (MRSA) biofilm. More recently, a study looking at the antibiotic resistant polymicrobial biofilms of Pseudomonas aeruginosa and MRSA in a maxillary sinus model were treated with a methylene blue/ethylenediamine tetraacetic acid (EDTA) photosensitizer and 670-nm non-thermal activating light. The results demonstrated that aPDT reduced the CRS polymicrobial biofilm by $>99.99 \%$ after a single treatment. Multiple benefits could potentially derive from the utilization of non-pharmacological treatment of infections, particularly avoidance of microbial resistance [27].

Our study represents the first clinical experience of aPDT in a cohort of patients with refractory CRS. The most common diagnosis in this group of patients was CRSsNP [\% 31.25], with CRSwNP including AFRS [\% 68.75] being the most prevalent subgroup in this category. Analyzing safety outcomes, we only encountered 3 mild and self-limiting adverse events during the study out of a total of 43 intervened sinuses: a stinging sensation in 2 patients and mild bleeding in one. Some of these may be explained by the fact that the balloons were hard to insert into some of the paranasal sinuses as the current version of the balloon catheter tip is fairly soft and requires some manipulation to insert into the maxillary and frontal sinuses. There is a slight learning curve for placement of the balloon catheter within the sinuses. Physician experience with the placement of the balloon likely plays an important role in comfort for the patient. Local anesthetic may be applied to avoid such discomfort and pain.

In terms of efficacy, the clinical response we obtained showed a significant reduction in endoscopic mucosal scores at 3 months. 
We observed some fluctuation of the mucosal scores including a worsening in 7 patients at the 6-month post-procedure visit, but these scores did not diminish to original baseline values. This might be explained by the fact that biofilm-mediated diseases often show a relapsing and remitting course and have variable growth rates with variations in clinical presentation [28]. Although pre and post-treatment mucosal membrane electron microscopy was not utilized, the enhancement in sinus score and reduction in purulent discharge is an indirect marker of efficacy of the treatment as well as improved post-treatment mucociliary clearance.

\section{Conclusion}

Patients with refractory CRS can be safely treated with aPDT on an outpatient basis. These early efficacy results, while promising, will require further validation in prospective clinical trials.

\section{Acknowledgement}

None.

\section{Conflict of Interest}

The authors declare that they have no competing interests. Light catheters and photosensitizer were supplied by Ondine Biomedical at no charge.

\section{References}

1. Lethbridge-Cejku M, Schiller JS, Bernadel L (2002) Summary health statistics for U.S. adults: National Health Interview Survey. Vital and health statistics Series 10, Data from the National Health Survey 2004: $1-151$.

2. Bhattacharyya N (2010) Ambulatory sinus and nasal surgery in the United States: demographics and perioperative outcomes. Laryngoscope 120(3): 635-638.

3. Meltzer EO, Hamilos DL (2011) Rhinosinusitis diagnosis and management for the clinician: a synopsis of recent consensus guidelines. Mayo Clin Proc 86(5): 427-443.

4. Desrosiers M (2004) Refractory Chronic Rhinosinusitis: Pathophysiology and Management of Chronic Rhinosinusitis Persisting After Endoscopic Sinus Surgery. Curr Allergy Asthma Rep 4: 200-207.

5. Rudmik L, Smith TL (2011) Quality of life in patients with chronic rhinosinusitis. Curr Allergy Asthma Rep 11(3): 247-252.

6. Luke Rudmik L, Smith TL, Schlosser RJ, Hwang PH, Mace JC, et al. (2014) Productivity Costs in Patients with Refractory Chronic Rhinosinusitis. Laryngoscope 124(9): 2007-2012.

7. Ezzat WF, Fawaz SA, Rabie H, Hamdy TA, Shokry YA (2015) Effect of topical ofloxacin on bacterial biofilms in refractory post-sinus surgery rhino-sinusitis. Eur Arch Otorhinolaryngol 272(9): 2355-2361.

8. Al-Qudah M (2011) Refractory Chronic Rhinosinusitis: Etiology \& Management. In Peculiar Aspects of Rhinosinusitis. Gian Luigi Marseglia, Davide Paolo Caimmi (Eds,). In Tech Open.

9. Cohen M, Kofonow J, Nayak JV, Palmer JN, Chiu AG, et al. (2009) Biofilms in chronic rhinosinusitis: A review. Am J Rhinol Allergy 23(3): 255-260.

10. Palmer JN (2005) Bacterial biofilms: Do they play a role in chronic sinusitis? Otolaryngol Clin North Am 38(6): 1193-1201, viii.
11. Zhang Z, Adappa ND, Chiu AG, Doghramji LJ, Cohen NA, et al. (2015) Biofilm forming bacteria and quality of life improvement after sinus surgery. Int Forum Allergy Rhinol 5(7): 643-649.

12. Costerton W, Veeh R, Shirtliff M, Pasmore M, Post C, et al. (2003) The application of biofilms science to the study and control of chronic bacterial infections. J Clin Invest 112(10): 1466-1477.

13. Konopka K, Goslinski T (2007) Photodynamic therapy in dentistry. J Dent Res 86(8): 694-707.

14. Morley S, Griffiths J, Philips G, Moseley H, O'Grady C, et al. (2012) Phase Ila randomised, placebo-controlled study of antimicrobial photodynamic therapy in bacterially colonised, chronic leg ulcers and diabetic foot ulcers. A new approach to antimicrobial therapy. Br J Dermatol 168(3): 617-624.

15. Triesscheijn M, Baas P, Schellens JH, Stewart FA (2006) Photodynamic therapy in oncology. Oncologist 11(9): 1034-1044.

16. Agostinis P, Berg K, Cengel KA, Foster T, Girotti A, et al. (2011) Photodynamic therapy of cancer: an update. CA Cancer J Clin 61(4): 250-281.

17. Maccormack MA (2008) Photodynamic therapy in dermatology: an update on applications and outcomes. Semin Cutan Med Surg 27(1): 5262.

18. Soergel P, Hillemanns P (20120) Photodynamic therapy for intraepithelial neoplasia of the lower genital tract. Photodiagnosis Photodynamic Theraphy 7(1): 10-14.

19. Kharkwal GB, Sharma SK, Huang YY, Dai T, Hamblin MR (2011) Photodynamic Therapy for Infections: Clinical Applications. Lasers in Surgery and Medicine 43(7): 755-767.

20. Dai T, Huang YY, Hamblin MR (2009) Photodynamic therapy for localized infections--state of the art. Photodiagnosis Photodyn Ther 6(3-4): 170188.

21. Biel MA, Sievert C, Usacheva M, Teichert M, Balcom J (2011) Antimicrobial photodynamic therapy treatment of chronic recurrent sinusitis biofilms. Int Forum Allergy Rhinol 1(5): 329-334.

22. Krespi YP, Kizhner V (2011) Phototherapy for chronic rhinosinusitis. Lasers Surg Med 43: 187-191

23. Bashkatov AN, Genina ÉA, Chikina EÉ (2005) Laser therapy of acute and chronic maxillary sinusitis. Proc SPIE 6344 Advanced Laser Technologies.

24. Bent JP $3^{\text {rd }}$, Kuhn FA (1994) Diagnosis of allergic fungal sinusitis. Otolaryngol Head Neck Surg 111(5): 580-588.

25. Brook I (2005) The role of bacteria in chronic rhinosinusitis. Otolaryngol Clin North Am 38: 1171-1192.

26. Lanza DC, Dhong H-J, Tantilipikorn P, Tanabodee J, Nadel DM, et al. (2006) Fungus and Chronic Rhinosinusitis: From Bench to Clinical Understanding. Ann Otol, Rhinol Laryngol suppl: 27-34.

27. Vohra F, Akram Z, Safii SH, Vaithilingam RD, Ghanem A, et al. (2016) Role of antimicrobial photodynamic therapy in the treatment of aggressive periodontitis: A systematic review. Photodiagnosis Photodyn Ther 13: 139-147.

28. Foreman A, Psaltis AJ, Wai Tai L, Wormald PJ (2009) Characterization of bacterial and fungal biofilms in chronic rhinosinusitis. Am J Rhinol Allerg 23(6): 556-561. 